\title{
Research on optimization design of conformal cooling channels in hot stamping tool based on response surface methodology and multi- objective optimization
}

\author{
Bin $\mathrm{He}^{1}$, Yanglei $\mathrm{Si}^{1}$, Liang Ying ${ }^{1}$ and Ping $\mathrm{Hu}^{1, \mathrm{a}}$ \\ ${ }^{1}$ School of Automotive Engineering, Faculty of Vehicle Engineering and Mechanics, State Key Laboratory of Structural Analysis for Industrial \\ Equipment, Dalian University of Technology, Dalian 116024, P.R. China
}

\begin{abstract}
In order to optimize the layout of the conformal cooling channels in hot stamping tools, a response surface methodology and multi-objective optimization technique are proposed. By means of an Optimal Latin Hypercube experimental design method, a design matrix with 17 factors and 50 levels is generated. Three kinds of design variables, the radius $R a d$ of the cooling channel, the distance $H$ from the channel center to tool work surface and the ratio rat of each channel center, are optimized to determine the layout of cooling channels. The average temperature and temperature deviation of work surface are used to evaluate the cooling performance of hot stamping tools. On the basis of the experimental design results, quadratic response surface models are established to describe the relationship between the design variables and the evaluation objectives. The error analysis is performed to ensure the accuracy of response surface models. Then the layout of the conformal cooling channels is optimized in accordance with a multiobjective optimization method to find the Pareto optimal frontier which consists of some optimal combinations of design variables that can lead to an acceptable cooling performance.
\end{abstract}

\section{Introduction}

Hot stamping technology which is widely applied in the automotive components manufacture provides the possibility to produce components with complex shape, high strength and less springback [1,2]. Cooling channels arranged inside hot stamping tools play an important role in the cooling efficiency and temperature uniformity of tools [3].

There have been a few researches about design and optimization of cooling channels. Straight hole design is the most common method which has been discussed by Hoffmann and Steinbeiss, and genetic algorithm was submitted to optimize the cooling system [4,5]. Liu et al. proposed a novel approach to design and manufacture the channels which were pre-embedded in a casting mould with pre-bending stainless pipes [6]. Sachs et al. first put forward the concept of conformal cooling channel design and produce the tool by 3D printing technology [7]. Xu et al. presented a systematic approach for conformal design which is evaluated by five criteria, including transient heat transfer condition, maximum distance from the mould surface to cooling channel, pressure drop, temperature drop and mould strength [8]. Park and Dang designed a cooling layout with an array of baffles to achieve the conformal characteristics [9]. Hu et al. raised four types of conformal designs and the longitudinal conformal cooling channel was proved as the optimal cooling design when inlet mass flow rate was large [10]. Wang et al. created a quadratic response surface model with sample data from Box-Behnken design and then optimized the regression model by a constrained particle swarm optimization method [11]. Jarrett et al. integrated GAMBIT and FLUENT by a MATLAB optimization program package to optimize the cooling layout in battery cells $[12,13]$. Although the conformal design was studied in many fields, the application for the optimal cooling layout of hot stamping tools has not yet been investigated.

In this paper, the radius of cooling channel, the distance from the channel center to tool work surface and the ratio of each channel center are considered as the design variables which can determine the layout of cooling channels. The average temperature and temperature deviation of work surface are chosen as evaluation indicators to assess the cooling performance. Based on the OLH experimental results, response surface models are established. Finally, the layout of cooling channels is optimized by multi-objective method and a Pareto frontier is obtained which reveals the optimal variables combination.

\section{Conformal cooling channel design}

During hot stamping process, the steel sheet is heated up to the austenite temperature range in a furnace until a

\footnotetext{
${ }^{\mathrm{a}}$ Corresponding author: pinghu@dlut.edu.cn
} 
homogeneous austenitic microstructure is obtained. Then the steel sheet is transferred to the press and subsequently formed and simultaneously quenched in the closed tools with a cooling system to produce the intended shape. The components finally exhibit a martensitic microstructure with $1500 \mathrm{MPa}$ tensile strength [14]. Straight hole design is the traditional cooling configuration employed in hot stamping tools [15].

\subsection{Comparative analysis of traditional and conformal design}

\subsubsection{Design structure}

Take an electric vehicle B-pillar punch tool as an example, different cooling structures of straight hole design and conformal cooling channel design are illustrated in Figure 1. The cooling water first flows into the water manifold and then passes into the three inserts and finally flows out from the outlet on the manifold. Obviously, the main difference of cooling design occurs in the middle insert which has a curved shape. The straight hole design is realized only through drilling intersectant holes on two edge surfaces and it is a nonisometric design that will lead to inhomogeneous cooling performance. On the contrary, the conformal cooling channel design is an isometric design which can achieve better cooling performance.

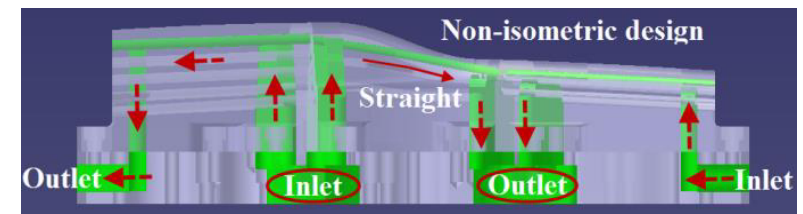

(a) Straight hole design

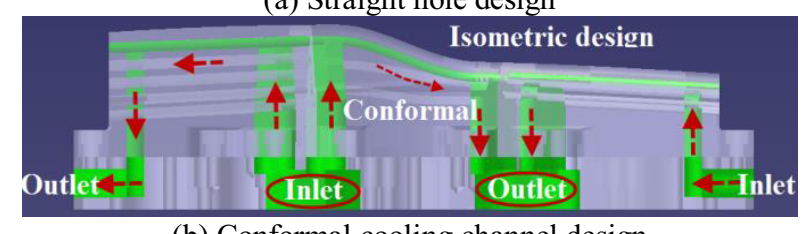

(b) Conformal cooling channel design

Figure 1. Structures of straight hole design and conformal cooling channel design.

\subsubsection{Cooling performance}

In order to prove the advantages of conformal cooling channel design, a heat transfer simulation is performed which can approximately describe the hot stamping process. Generally, the temperature distribution after achieving steady state is extracted as evaluation indicator and six stamping cycles are enough for hot stamping tool to reach a steady temperature fluctuation.

Table 1. Material property of tool.

\begin{tabular}{|l|l|}
\hline Parameter & Value \\
\hline Density $/ \mathrm{kg} \cdot \mathrm{m}^{-3}$ & 7900 \\
\hline
\end{tabular}

\begin{tabular}{|l|l|}
\hline $\begin{array}{l}\text { Thermal } \\
\text { conductivity / } \\
\mathrm{W} \cdot \mathrm{m}^{-1} \cdot \mathrm{K}^{-1}\end{array}$ & $-3 \times 10^{-5} \mathrm{~T}^{2}+5.7 \times 10^{-3} \mathrm{~T}+42.624$ \\
\hline $\begin{array}{l}\text { Special heat } / \mathrm{J} \cdot \mathrm{kg}^{-} \\
1 \cdot \mathrm{K}^{-1}\end{array}$ & 450 \\
\hline $\begin{array}{l}\text { Initial tool } \\
\text { temperature } /{ }^{\circ} \mathrm{C}\end{array}$ & 20 \\
\hline Mesh type & $\begin{array}{l}\text { DC3D4: a 4-node linear heat } \\
\text { transfer tetrahedron. }\end{array}$ \\
\hline
\end{tabular}

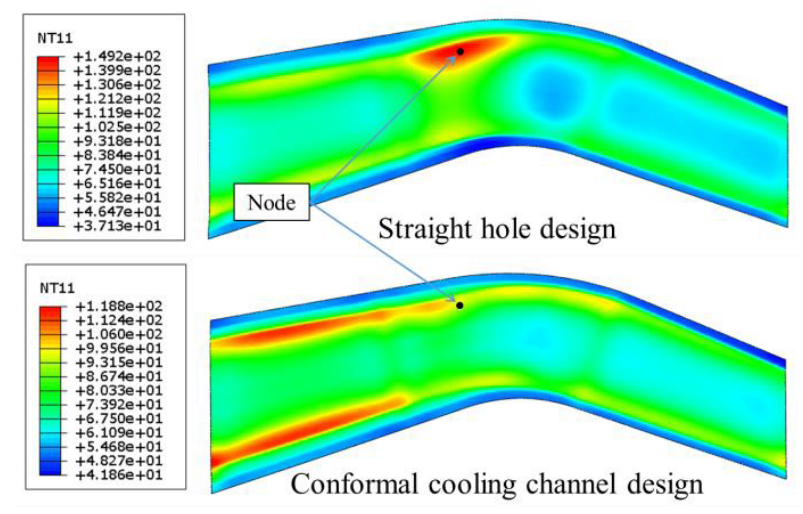

Figure 2. Temperature distributions of straight hole design and conformal cooling channel design.

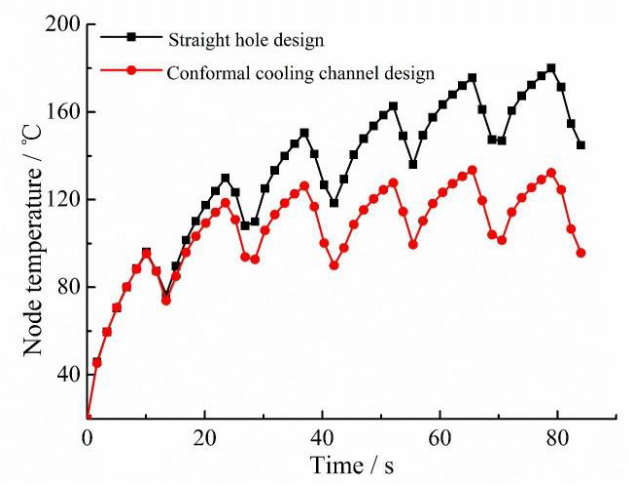

Figure 3. Temperature history of a node on the tool rounded corner.

The tool material is H13 steel. Its material property is listed in Table 1. Supposing that the total time of a stamping cycle is $14 \mathrm{~s}$ and among that the stamping and holding time, feeding time and reclaiming time are $10 \mathrm{~s}, 2$ $\mathrm{s}, 2 \mathrm{~s}$ respectively. The time-averaged heat flux of $190,000 \mathrm{~W} \cdot \mathrm{m}^{-2}$ is loaded on the work surface to simulate the stamping and quenching stage, and it is regarded as 0 $\mathrm{W} \cdot \mathrm{m}^{-2}$ during the feeding and reclaiming stage. The smooth process by high order polynomial is implemented in the transition of heat flux load at different stages. According to the empirical equations and limit size of cooling channels, the convectional heat transfer coefficient between the channel wall and the cooling water is set as a constant of $5,417 \mathrm{~W} \cdot \mathrm{m}^{-2} \cdot \mathrm{K}^{-1}$.

It is noted from Figure 2 that the same FEM model and boundary conditions are used, and for the straight hole design, the maximum work surface's temperature is $149.2{ }^{\circ} \mathrm{C}$, but for the conformal cooling channel design, that is reduced to $118.8{ }^{\circ} \mathrm{C}$. Besides, the temperature uniformity is obviously improved and especially shown 
in the middle insert. Due to the convex shape, the temperature is always concentrated on the rounded corner. In Figure 3, it is found after the first stamping cycle that the temperature at the specific node of straight hole design is always higher than that of conformal cooling channel design. Moreover, it is obvious that the tool using conformal cooling channel design takes less time to achieve temperature stability. The present study will focus on how to obtain the optimal layout of cooling channels with conformal design.

\subsection{Experimental design by using Optimal Latin Hypercube method}

\subsubsection{Model verification}

The accuracy of the FEM simulation generally increases with the increase of mesh number. But at the same time, the computing time is also greatly increased especially for optimization process which requires hundreds of computations. Therefore, before optimization, the FEM model should be verified with a mesh independence analysis to ascertain a mesh size that would achieve convincible results in an acceptable computing time.

Take the middle insert as an example, which is more sensitive to design structure, the same material property and boundary conditions are set up and different models with a mesh range of $2 \mathrm{~mm}$ to $25 \mathrm{~mm}$ are simulated. The relative error of work surface's temperature and the computing time for the FEM models with different mesh base sizes are shown in Figure 4.

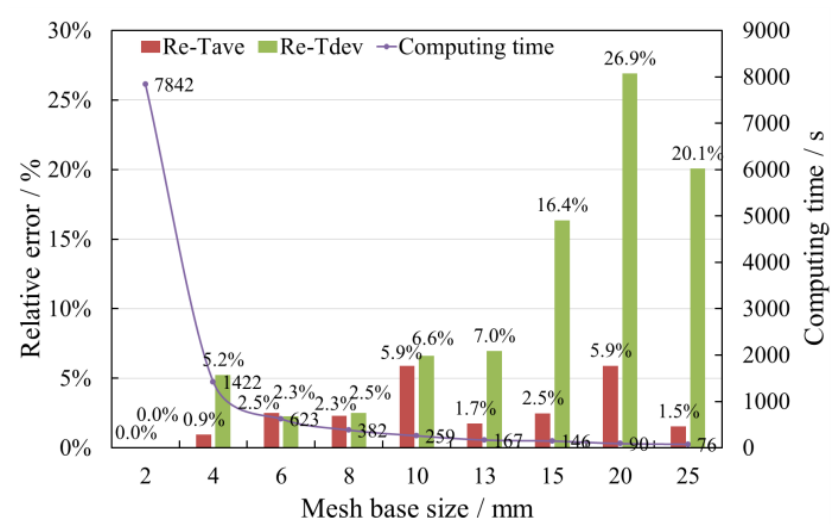

Figure 4. Relative error of work surface's temperature and computing time for FEM models with different mesh base sizes.

The Re-Tave and Re-Tdev in the Figure 4 stand for the relative error of average temperature and temperature deviation on the work surface comparing the model with $2 \mathrm{~mm}$ mesh base size. It is demonstrated that Re-Tdev increases with the increase of mesh base size but the ReTave presents random fluctuation. It is not surprising that the computing time reduces with the increase of mesh base size and the computing efficiency is improved sharply for the mesh size of $2 \mathrm{~mm}$ to $4 \mathrm{~mm}$. Based on the independence analysis, it is easy to find that the optimal mesh base size is $8 \mathrm{~mm}$ for the present problem.

\subsubsection{Range of design variables}

The definition of design variables is depicted in Figure 5. It is noted that Rad symbol denotes the radius of the cooling channel, $H$ symbol refers to the distance from the channel center to tool work surface and rat symbol stands for the ratio of each channel center.

Considering the design and assembly constraints, the design variables including the radius, distance and ratio must be restricted to a range to avoid the intersection and interference. Whether in the experimental design or in the optimization process, design variables always change within the range set out in Table 2.

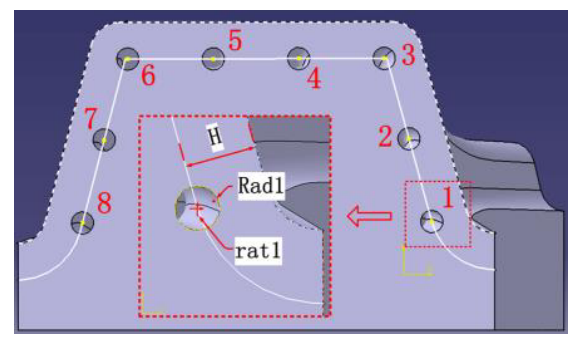

Figure 5. Schematic diagram of design variables.

Table 2. Range of design variables.

\begin{tabular}{|l|l|l|l|}
\hline Design variables & Lower value & Upper value & Unit \\
\hline$H$ & 13 & 17 & $\mathrm{~mm}$ \\
\hline Rad $1 \sim \operatorname{Rad} 8$ & 4 & 6 & $\mathrm{~mm}$ \\
\hline rat 1 & 0.05 & 0.127 & - \\
\hline rat 2 & 0.203 & 0.237 & - \\
\hline rat3 & 0.313 & 0.347 & - \\
\hline rat 4 & 0.423 & 0.457 & - \\
\hline rat5 & 0.533 & 0.567 & - \\
\hline rat6 & 0.643 & 0.677 & - \\
\hline rat7 & 0.753 & 0.787 & - \\
\hline rat 8 & 0.863 & 0.95 & - \\
\hline
\end{tabular}

\subsubsection{Design matrix}

Design of experiment (DOE) is an approach widely applied in industry that can reveal the relationship of design variables and response using a small amount of sample points. The Optimal Latin Hypercube is a method of DOE in which the number of levels of each factor is equal to the number of design points evenly spread within an n-dimensional space defined by the $n$ factors through combination optimization. A design matrix with 17 factors i.e. design variables and 50 levels displayed in Table 3 is generated by OLH method. Due to space limitations, only some design points are listed in Table 3. The design variables adhere to the range placed in Table 2. The type of $H$ and $\operatorname{Rad} 1 \sim \operatorname{Rad} 8$ is integer, but rat $1 \sim$ rat8 is real.

Table 3. Design matrix generated by OLH method.

\begin{tabular}{|l|l|l|l|l|l|}
\hline $\begin{array}{l}\text { Design } \\
\text { variables }\end{array}$ & Des.1 & Des.2 & $\begin{array}{l}\text { Des.3 } \\
\text { Des.48 }\end{array}$ & Des.49 & Des.50 \\
\hline$H$ & 14 & 14 & $\ldots$ & 15 & 14 \\
\hline Rad 1 & 5 & 4 & $\ldots$ & 5 & 6 \\
\hline Rad 2 & 4 & 5 & $\ldots$ & 5 & 5 \\
\hline
\end{tabular}




\begin{tabular}{|l|l|l|l|l|l|}
\hline Rad3 & 5 & 5 & $\ldots$ & 5 & 6 \\
\hline Rad4 & 6 & 6 & $\ldots$ & 5 & 5 \\
\hline Rad5 & 5 & 4 & $\ldots$ & 6 & 5 \\
\hline Rad6 & 5 & 5 & $\ldots$ & 4 & 6 \\
\hline Rad7 & 5 & 5 & $\ldots$ & 4 & 6 \\
\hline Rad 8 & 5 & 4 & $\ldots$ & 5 & 5 \\
\hline rat 1 & 0.0516 & 0.1066 & $\ldots$ & 0.1019 & 0.1003 \\
\hline rat 2 & 0.208 & 0.209 & $\ldots$ & 0.211 & 0.207 \\
\hline rat3 & 0.324 & 0.343 & $\ldots$ & 0.332 & 0.340 \\
\hline rat4 & 0.429 & 0.424 & $\ldots$ & 0.450 & 0.440 \\
\hline rat5 & 0.563 & 0.543 & $\ldots$ & 0.534 & 0.564 \\
\hline rat6 & 0.661 & 0.661 & $\ldots$ & 0.674 & 0.665 \\
\hline rat7 & 0.785 & 0.774 & $\ldots$ & 0.756 & 0.754 \\
\hline rat8 & 0.8648 & 0.9269 & $\ldots$ & 0.9447 & 0.934 \\
\hline
\end{tabular}

\subsection{Response surface model establishment}

\subsubsection{Samples from $\mathrm{OLH}$}

The sample points in the design matrix are executed automatically by an integration flow established by ISIGHT which can transfer the data information among the CATIA, ABAQUS and data process model. The workflow is shown in Figure 6.

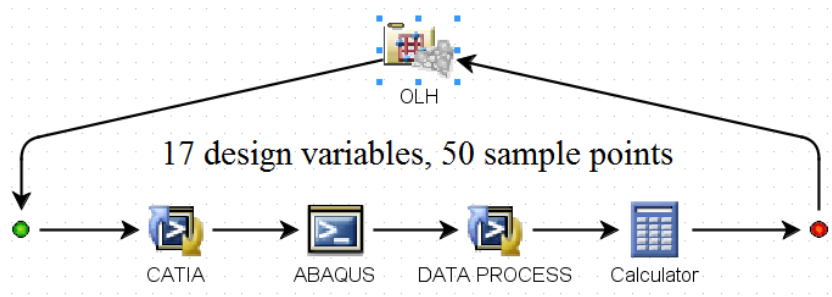

Figure 6. Workflow of OLH process.

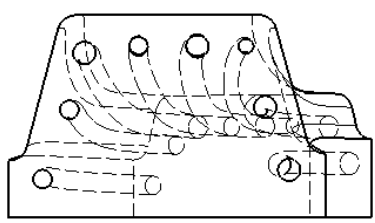

Design 3

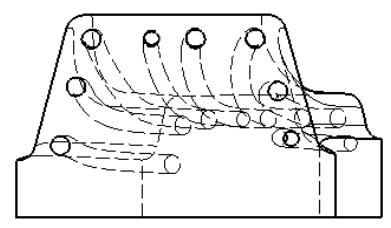

Design 47

Figure 7. CAD models of Des. 3 and Des. 47.

The parameterized CAD model is created by CATIA, and then the CAD routine is mapped to ABAQUS. Next the temperature data simulated by ABAQUS are extracted to perform the data process. The response, i.e. evaluation indicators are solved in the Calculator. According to the design matrix including 17 design variables and 50 sample points, the workflow will auto run 50 times in total and the response values corresponding to the 50 sample points are listed in Table 4.

Tave and Tdev are the average temperature and temperature uniformity of work surface, respectively. It is noted that Tave and Tdev are different for different

designs. In order to show more images to design differences, two different designs are illustrated in Figure 7.

Table 4. Response values corresponding to the 50 sample points.

\begin{tabular}{|l|l|l|l|l|l|}
\hline $\begin{array}{l}\text { Sample } \\
\text { points }\end{array}$ & Tave & Tdev & $\begin{array}{l}\text { Sample } \\
\text { points }\end{array}$ & Tave & Tdev \\
\hline Des.1 & 71.93 & 11.97 & Des.26 & 75.94 & 14.78 \\
\hline Des.2 & 70.95 & 12.18 & Des.27 & 78.09 & 14.90 \\
\hline Des.3 & 85.95 & 17.63 & Des.28 & 86.38 & 17.32 \\
\hline Des.4 & 83.38 & 16.89 & Des.29 & 75.98 & 14.59 \\
\hline Des.5 & 78.80 & 15.23 & Des.30 & 66.74 & 10.61 \\
\hline Des.6 & 79.10 & 15.85 & Des.31 & 78.56 & 14.56 \\
\hline Des.7 & 71.57 & 11.15 & Des.32 & 65.91 & 10.48 \\
\hline Des.8 & 70.26 & 12.02 & Des.33 & 66.74 & 11.50 \\
\hline Des.9 & 73.60 & 11.95 & Des.34 & 63.15 & 10.08 \\
\hline Des.10 & 72.98 & 14.48 & Des.35 & 75.41 & 14.78 \\
\hline Des.11 & 72.48 & 13.92 & Des.36 & 84.59 & 15.26 \\
\hline Des.12 & 76.13 & 13.55 & Des.37 & 85.82 & 17.53 \\
\hline Des.13 & 84.08 & 16.16 & Des.38 & 70.63 & 13.38 \\
\hline Des.14 & 82.31 & 15.66 & Des.39 & 74.66 & 13.95 \\
\hline Des.15 & 71.00 & 13.12 & Des.40 & 81.59 & 14.81 \\
\hline Des.16 & 77.89 & 12.65 & Des.41 & 68.84 & 10.60 \\
\hline Des.17 & 79.48 & 14.32 & Des.42 & 78.89 & 15.62 \\
\hline Des.18 & 71.12 & 12.69 & Des.43 & 68.64 & 10.39 \\
\hline Des.19 & 83.65 & 15.80 & Des.44 & 77.12 & 14.05 \\
\hline Des.20 & 85.44 & 17.29 & Des.45 & 73.15 & 12.57 \\
\hline Des.21 & 71.12 & 13.05 & Des.46 & 70.35 & 13.00 \\
\hline Des.22 & 85.23 & 15.03 & Des.47 & 65.70 & 9.87 \\
\hline Des.23 & 67.80 & 10.89 & Des.48 & 70.21 & 13.51 \\
\hline Des.24 & 82.14 & 16.18 & Des.49 & 75.63 & 15.12 \\
\hline Des.25 & 80.80 & 16.00 & Des.50 & 70.43 & 11.09 \\
\hline
\end{tabular}

\subsubsection{Model fitting and error analysis}

Response surface methodology (RSM) is a statistical and mathematical technique for relationship evaluation between design variables and response variables based on Taylor's expansion. The quadratic polynomial model can be expressed as,

$$
y=\beta_{0}+\sum \beta_{i} x_{i}+\sum \beta_{i i} x_{i}^{2}+\sum \beta_{i j} x_{i} x_{j}+\varepsilon
$$

where $\beta_{0}$ is constant term, $\beta_{i}$ is linear coefficient, $\beta_{i i}$ is quadratic coefficient, $\beta_{i j}$ is interaction coefficient, $i, j$ are the number of design variables, and $\varepsilon$ is statistical error.

Depending on the design variables in Table 3 and the response variables in Table 4, the regression response surface models of Tave and Tdev are expressed as Eq. (2) and Eq. (3). Both of the two mathematical models described Tave and Tdev contain 25 terms. Tave model is composed by 1 constant term, 9 linear terms, 6 quadratic 
terms and 9 interactive terms. Tdev model is composed by 1 constant term, 8 linear terms, 9 quadratic terms and 7 interactive terms.

$$
\begin{aligned}
& \text { Tave }=873.766-86.03 H+9.04 R a d 1-0.37 \operatorname{Rad} 2 \\
& -1.08 \text { Rad } 5+10.59 \text { Rad } 8+137.11 \text { rat } 1+6099.08 \text { rat } 4 \\
& -5885.66 \mathrm{rat} 5+41.51 \mathrm{rat} 7+0.16 H^{2}-0.68 \operatorname{Rad}^{2}- \\
& \text { 1526.11rat } 2^{2}-6939.08 \text { rat } 4^{2}+4336.8 \text { rat }^{2}-662.83 \\
& \operatorname{rat}^{2}-0.62 H * \operatorname{Rad} 1-0.11 H * \operatorname{Rad} 3-0.03 H * \\
& \operatorname{Rad} 4-0.06 H * \operatorname{Rad} 7-0.32 H * \operatorname{Rad} 8-10.18 H * \\
& \text { rat } 1+44.25 H * \text { rat } 2+77.99 H * \text { rat } 5+60.29 H * \text { rat } 6 \\
& T d e v=-919.38+0.25 \operatorname{Rad} 1-3.47 \operatorname{Rad} 5-2.23 \operatorname{Raad} 7 \\
& +1563.37 \text { rat } 2+2451.49 \text { rat } 4-895.89 \text { rat } 5-2381.71 \\
& \operatorname{rat} 6+3201.53 \operatorname{rat} 7-0.24 \operatorname{Rad} 3^{2}+0.21 \operatorname{Rad} 7^{2}- \\
& 245.01 \text { rat } 1^{2}-3609.53 r a t 2^{2}-2775.08 r a t 4^{2}+ \\
& 675.75 \text { rat }^{2}+1825.76 \text { rat }^{2}-1978.71 \text { rat }^{2}+ \\
& 4.91 \mathrm{rat}^{2}+0.11 H * \operatorname{Rad} 3+0.22 H * \operatorname{Rad} 5- \\
& 0.04 H * \operatorname{Rad} 6+2.67 H * \operatorname{rat} 1+0.61 H * \text { rat } 3+ \\
& 10.16 H * \text { rat } 5-7.76 H * \text { rat } 7
\end{aligned}
$$

The cross validation method is used for error analysis (Figure 8) in this study. It is noted that match of the models are good and reliable, and the adjusted $R$ square coefficient of Tave is 0.94 and Tdev is 0.95 . It means that the two models present a good characterization of the relationship between design variables and response variables.

\subsubsection{Pareto graph analysis}

Pareto graph reflects the contribution degree of all items in the fitting model for each response. Figure 9 shows the Pareto graph analysis of Tave and Tdev. Red bar stands for negative effect, and blue bar represents positive effect. It is noted that whether for Tave or Tdev, the design variables of rat have the most significant effect. The effect of $H$ is smaller than rat but bigger than all Rad variables. For Tave, rat 4 and rat 5 show significant impact and the effect degrees reach up to $35 \%$ and $39 \%$ respectively. However, effect degrees of other variables are all below $11 \%$. For Tdev, effect degrees of rat 7 , rat 4 and rat 2 are $28 \%, 24 \%$ and $23 \%$ respectively. Similarly, effect degrees of other variables are all under $11 \%$.

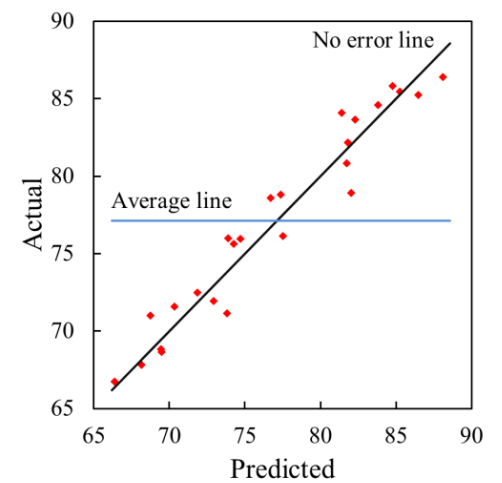

(a) Error analysis of Tave

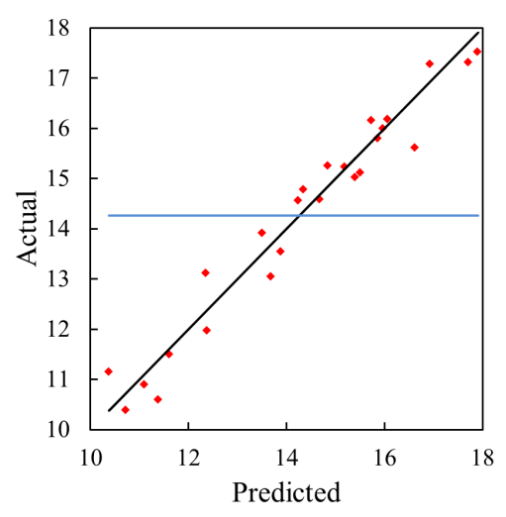

(b) Error analysis of Tdev

Figure 8. Error analysis of Tave and Tdev.

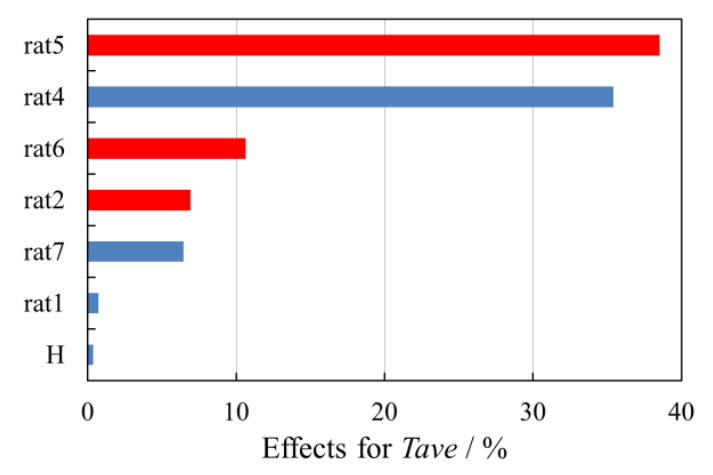

(a) Pareto graph of Tave

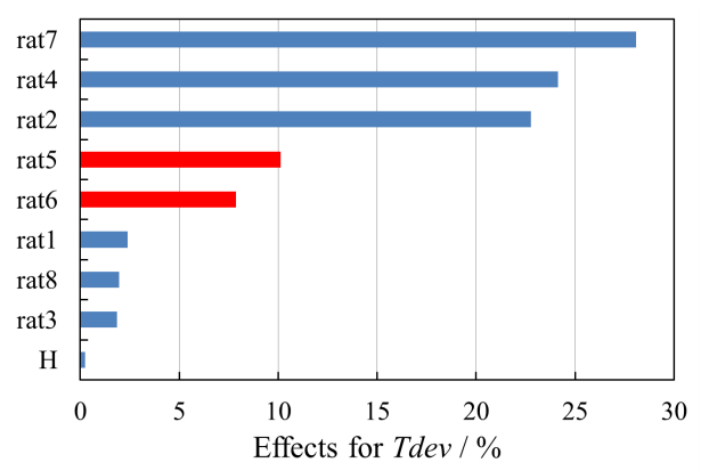

(b) Pareto graph of Tdev

Figure 9. Pareto graph analysis of Tave and Tdev.

\section{Optimization}

\subsection{Model and process}

Generally, Tave and Tdev are two conflicting response variables. When the average temperature attains the minimum value, the temperature uniformity is more obvious, and vice versa. Therefore, the multi-objective optimization method which will compromise with the two objectives is utilized to explore the optimal layout of cooling channels. The mathematical description of the optimization problem is given as, 


$\begin{array}{ll}\text { Minimize } & \text { Tave Eq. }(2) \\ \text { Minimize } & \text { Tdev Eq. }(3) \\ \text { Variable range } & \{H, \operatorname{Rad} 1-\text { Rad } 8, \text { rat } 1-\text { rat } 8\} \\ & \text { abide by Table } 2\end{array}$

NSGA-II (Non-dominated Sorting Genetic Algorithm) which is well-suited for highly non-linear and discontinuous design spaces is used in the present study. By this method, each objective is treated separately. Standard genetic operation of mutation and crossover is performed on the designs. Selection process is based on two main mechanisms, "non-dominated sorting" and "crowding distance sorting". The population size which controls the number of solutions generated at each iteration is set to 400 and the number of generations which controls the number of iterations that the algorithm will execute before termination is 40 . The total number of function evaluations exhausted by the algorithm equals the product of the population size and the number of generations. The process model created in ISIGHT is shown in Figure 10. The design variables of Des. 1 from OLH design matrix are used to initialize the optimization model.

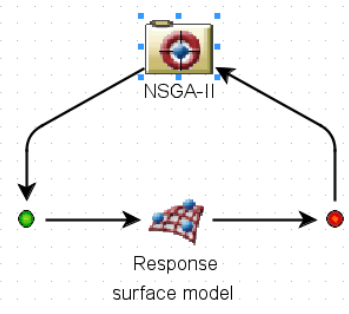

Figure 10. Optimization process.

\subsection{Pareto frontier and feasibility analysis}

A total of 16,000 evaluations are operated for objective functions. Due to the hot stamping simulation has been regressed as mathematical models by OLH and response surface method before optimization, the optimization efficiency is improved greatly and only $5 \mathrm{~min}$ is spent. A total of 329 Pareto-optimal solutions are obtained and the Pareto frontier results from Pareto-optimal solutions which present a convex shape is displayed in Figure 11. It is noted that after multi-objective optimization Tave will be controlled within the range of $54{ }^{\circ} \mathrm{C}$ to $58{ }^{\circ} \mathrm{C}$, and Tdev is kept from $3.5^{\circ} \mathrm{C}$ to $6.0{ }^{\circ} \mathrm{C}$. The Pareto solutions are uniformly continuous and the trend line of these solutions is a quadratic polynomial. The point 1 marked as black in Figure 11 is the optimal solution revealed by ISIGHT. The $H, R a d 1 \sim \operatorname{Rad} 8$ and rat $1 \sim \operatorname{rat} 8$ of point 1are, in order, 13, 4, 6, 6, 6, 6, 6, 6, 6, 0.127, 0.237, 0.313, $0.423,0.562,0.677,0.753,0.8631$. According to the Pareto solutions, it can be found that design variables of $H$ and $R a d 1 \sim \operatorname{Rad} 8$ tend to unique peak values, but rat $1 \sim$ rat 8 have more options. It is a further validation that design variables of rat $1 \sim \operatorname{rat} 8$ have more effect on response variables.

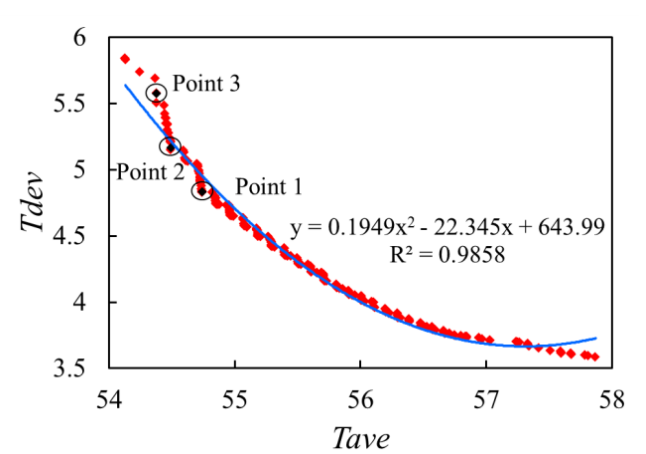

Figure 11. Pareto frontier graph.

In order to verify the accuracy of the optimization results, the design variables of three points including point 1, 2 and 3 are taken back to CAD routine and FEM model to carry out the hot stamping simulation separately. Tave and Tdev values getting from simulation and mathematical model are shown in Table 5. The results demonstrate that the optimization using regress models are not accurate enough comparing with direct simulation. But relatively speaking, Tave obtained by model optimization, which is always less than $15 \%$, is more accurate than Tdev. The relative error of Tdev is below $45 \%$, which means that though the $R$ square of Tdev model reaches up to 0.95 , the design space is still not entirely covered by the regress model. By increasing sample points or adjusting the experimental design process, the prediction precision and optimization accuracy will be improved. Although the prediction error is inevitable when using the response surface model to perform optimization, this method has a high costeffective compared to the direct simulation optimization which represents a high computational cost.

Table 5. Feasibility analysis of the optimization results.

\begin{tabular}{|l|l|l|l|l|l|l|}
\hline \multirow{2}{*}{} & \multicolumn{2}{|c|}{ Point 1 } & \multicolumn{2}{c|}{ Point 2 } & \multicolumn{2}{c|}{ Point 3 } \\
\cline { 2 - 7 } & Tave & Tdev & Tave & Tdev & Tave & Tdev \\
\hline Simulation & 63.5 & 8.6 & 62.1 & 8.8 & 61.0 & 8.1 \\
\hline $\begin{array}{l}\text { Mathematical } \\
\text { model }\end{array}$ & 54.7 & 4.8 & 54.5 & 5.2 & 54.4 & 5.6 \\
\hline Relative error & $14 \%$ & $44 \%$ & $12 \%$ & $41 \%$ & $11 \%$ & $31 \%$ \\
\hline
\end{tabular}

The temperature distributions before and after optimization are illustrated in Figure 12. Des. 1 listed in Table 3 is used as the initial design before optimization and Point 1 is the optimal solution recommended by Pareto solutions in ISIGHT. Obviously, the temperature distribution including average temperature and temperature uniformity has been improved after optimization. Tave and Tdev are separately reduced by $11.7 \%$ and $28.2 \%$. Moreover, the design parameters of cooling channels after optimized become more uniform and helpful to manufacture the channels. The whole points on Pareto frontier are the optimal combinations of design variables, however, the design combinations which contribute to the convenience of processing and the low cost should be prioritized. 


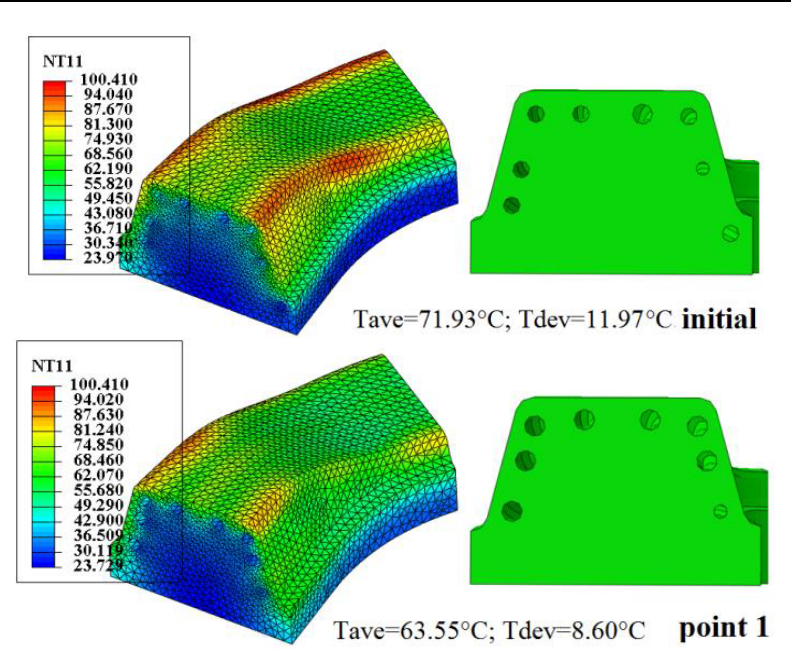

Figure 12. Temperature distribution before and after optimization.

\section{Advanced manufacture}

When adopting the conformal cooling channel design in hot stamping tools, how to realize the low-cost manufacture process is a tough issue. An advanced manufacture technology is proposed in this paper and its manufacturing process is displayed in Figure 13. The key step is to use the ceramic core model by extrusion to realize the channels' structure with conformal design. This advanced manufacture method is seen as a combination of ceramic core making and casting process.

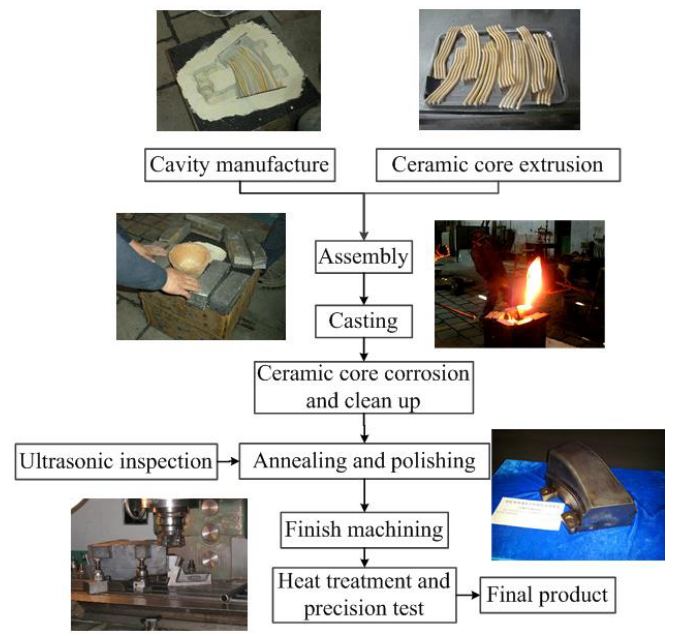

Figure 13. Manufacture process of hot stamping tools with conformal cooling channel design.

Look at Figure 13, first, the sand mould cavity and ceramic core with conformal shape are prepared. Second, the cavity, core, runner and riser are assembled into a gating system. Third, the casting process and the heat treatment after casted are performed. The survival state of ceramic core should be paid close attention in the casting process to prevent fracture. Fourth, after the cast mould cooling down, the ceramic core wrapped inside mould is cleaned by alkaline corrosion with a high temperature caldron. Fifth, ultrasonic inspection is used to find the severe defects, and if there are no serious defects, the casting mould will be annealed and polished subsequently, otherwise further evaluation of that mould will be carried out. Sixth, to operate the surface finishing and to quench hardened the casting mould. Finally, after a dimensional accuracy test, a finished hot stamping tool insert with conformal cooling channel design is produced successfully. By this method, the tool insert with any conformal structure is not difficult to be manufactured. Further verification tests of temperature distribution will be carried out in future studies.

\section{Acknowledgements}

This work was funded by the Key Project of the National Natural Science Foundation of China (No.11272075) and China Postdoctoral Science Foundation (2014M561223) and the Fundamental Research Funds for the Central Universities (DUT15TD31).

\section{References}

1. H. Karbasian, A.E. Tekkaya, J Mater Process Tech., 210 (2010) 2103-2118.

2. B. He, L. Ying, P. Hu, Y. Yu, X. Zhao, L.W. Zhang, Adv Mater Res., 1063 (2014) 186-189.

3. W. Lim, H. Choi, S. Ahn, B. Kim, Int J Adv Manuf Tech., 70 (2014) 1189-1203.

4. H. Hoffmann, H. So, H. Steinbeiss, CIRP AnnManuf Tech., 56 (2007) 269-272.

5. H. Steinbeiss, H. So, T. Michelitsch, H. Hoffmann, Production Engineering, 1 (2007) 149-155.

6. H. Liu, C. Lei, Z. Xing, Int J Adv Manuf Tech., 69 (2013) 211-223.

7. E. Sachs, E. Wylqnis, S. Allen, M. Cima, H. Guo, Polym Eng Sci., 40 (2000).

8. X. Xu, E. Sachs, S. Allen, Polym Eng Sci., 41 (2001).

9. H. Park, X. Dang, Int J Precis Eng Man., 11 (2010) 879-890.

10. P. Hu, B. He, L. Ying, Appl Therm Eng., 96 (2016) 338-351.

11. G. Wang, G. Zhao, H. Li, Y. Guan, Expert Syst Appl., 38 (2011) 6705-6719.

12. A. Jarrett, I.Y. Kim, J Power Sources, 196 (2011) 10359-10368.

13. A. Jarrett, I.Y. Kim, J Power Sources, 245 (2014) 644-655.

14. M. Merklein, J. Lechler, J Mater Process Tech., 177 (2006) 452-455.

15. F. Schieck, C. Hochmuth, S. Polster, A. Mosel, CIRP Journal of Manufacturing Science and Technology, 4 (2011) 189-199. 